\title{
Post-exercise changes in blood pressure, heart rate and rate pressure product at different exercise intensities in normotensive humans
}

C.L.M. Forjaz ${ }^{1}$, Y. Matsudaira', F.B. Rodrigues ${ }^{1}$, N. N unes ${ }^{1,2}$ and C.E. Negrão ${ }^{1,2,3}$

\author{
${ }^{1}$ Laboratório de Fisiologia do Exercício, Escola de Educação Física e Esportes, \\ ${ }^{2}$ Laboratório de Fisiologia Cardiovascular do Exercício, and \\ 3Unidade de Hipertensão, Instituto do Coração, Faculdade de Medicina, \\ Universidade de São Paulo, São Paulo, SP, Brasil
}

\section{Correspondence}

C.L.M. Forjaz

Laboratório de Fisiologia

do Exercício

Escola de Educação Física e

Esportes, USP

Av. Prof. Mello Moraes, 65

05508-900 São Paulo, SP

Brasil

Fax: +55-11-813-5921

E-mail: cforjaz@usp.br

Publication supported by FAPESP.

Received July 3, 1997

Accepted July 16, 1998

\section{Abstract}

To evaluate the effect of exercise intensity on post-exercise cardiovascular responses, 12 young normotensive subjects performed in a randomized order three cycle ergometer exercise bouts of $45 \mathrm{~min}$ at 30 , 50 and $80 \%$ of $\mathrm{VO}_{2}$ peak, and 12 subjects rested for $45 \mathrm{~min}$ in a nonexercise control trial. Blood pressure (BP) and heart rate (HR) were measured for $20 \mathrm{~min}$ prior to exercise (baseline) and at intervals of 5 to 30 (R5-30), 35 to 60 (R35-60) and 65 to 90 (R65-90) min after exercise. Systolic, mean, and diastolic BP after exercise were significantly lower than baseline, and there was no difference between the three exercise intensities. After exercise at $30 \%$ of $\mathrm{VO}_{2}$ peak, $\mathrm{HR}$ was significantly decreased at R35-60 and R65-90. In contrast, after exercise at 50 and $80 \%$ of $\mathrm{VO}_{2}$ peak, $\mathrm{HR}$ was significantly increased at R5-30 and R35-60, respectively. Exercise at 30\% of $\mathrm{VO}_{2}$ peak significantly decreased rate pressure $(\mathrm{RP})$ product $(\mathrm{RP}=\mathrm{HR}$ x systolic $\mathrm{BP}$ ) during the entire recovery period (baseline $=7930 \pm 314 v s \mathrm{R} 5-30$ $=7150 \pm 326, \mathrm{R} 35-60=6794 \pm 349$, and R65-90 = 6628 \pm 311 , $\mathrm{P}<0.05$ ), while exercise at $50 \%$ of $\mathrm{VO}_{2}$ peak caused no change, and exercise at $80 \%$ of $\mathrm{VO}_{2}$ peak produced a significant increase at R5-30 (7468 \pm 267 vs $9818 \pm 366, \mathrm{P}<0.05)$ and no change at R35-60 or R6590. Cardiovascular responses were not altered during the control trial. In conclusion, varying exercise intensity from 30 to $80 \%$ of $\mathrm{VO}_{2}$ peak in young normotensive humans did not influence the magnitude of post-exercise hypotension. However, in contrast to exercise at 50 and $80 \%$ of $\mathrm{VO}_{2}$ peak, exercise at $30 \%$ of $\mathrm{VO}_{2}$ peak decreased post-exercise HR and RP.
Key words - Exercise intensity

- Blood pressure

- Heart rate

- Rate pressure product 


\section{Introduction}

Although an acute bout of submaximal exercise can cause important cardiovascular changes, there are few and controversial published data on the effect of different exercise intensities on blood pressure, heart rate and rate pressure product (an index of myocardial oxygen consumption). An increased understanding of this issue has clinical implications, in addition to being important in prescribing exercise intensities for humans.

Most studies dealing with post-exercise blood pressure responses have demonstrated that exercise reduces blood pressure during the recovery period (1-19). Nevertheless, the magnitude and the time course of blood pressure changes after exercise are inconsistent. In normotensives, the magnitude ranges from no change $(4,8,14,15,20)$ to falls as great as $30 \mathrm{mmHg}$ (19). In hypertensive humans, Somers et al. (18) observed that blood pressure levels are significantly lower than preexercise levels only during the first hour of recovery, while Pescatello et al. (14) found a significant blood pressure fall up to $12 \mathrm{~h}$ after exercise. In relation to post-exercise heart rate responses, the results are also controversial and less conclusive. Some investigators $(2,4,5,7,10-12,15,19)$ have observed an increase in heart rate levels during the recovery period. In contrast, others have reported no change $(1,3,6,8,12,14,20)$ or even decreases $(9,13)$ in post-exercise heart rate responses. The experimental protocol, the initial levels of blood pressure, and the type, duration and intensity of exercise may explain, in part, these discrepancies in postexercise blood pressure and heart rate responses (21).

It has been well established that the magnitude of neural and hemodynamic responses during exercise is directly related to exercise intensity $(22,23)$. Thus, it is possible that different exercise intensities have also distinct effects on cardiovascular changes after exercise. Hagberg et al. (9), in a study of older hypertensive men, observed that hypotension after exercise performed at $70 \%$ of maximal oxygen consumption $\left(\mathrm{VO}_{2} \mathrm{max}\right)$ was greater and lasted longer than after exercise performed at $50 \%$ of $\mathrm{VO}_{2}$ max. Piepoli et al. (15) reported that maximal exercise provoked an increase, while low to moderate exercise caused no change in post-exercise heart rate levels in normotensive subjects.

Because blood pressure and heart rate responses during the recovery period can be influenced by exercise intensity, it is possible that different intensities of exercise may also have distinct effects on post-exercise rate pressure product. Therefore, the goal of the present investigation was to study the effect of different exercise intensities on post-exercise blood pressure, heart rate and rate pressure product in humans.

\section{Material and Methods}

\section{Subjects}

Twelve healthy young normotensive subjects (4 women and 8 men; exercised subjects) were studied in the exercise trials, and twelve subjects ( 7 women and 5 men; rested subjects) with similar physical and cardiovascular characteristics were studied in the non-exercise control trial (Table 1). Their healthy condition was confirmed by physical examination and a maximal exercise test. None of the subjects engaged in any regular physical activity program. All procedures were approved by the General Hospital Ethics Committee, University of São Paulo, and all subjects gave informed written consent to participate in the study.

\section{Blood pressure measurements}

After a 5-min rest in the sitting position, blood pressure was measured three times during two different visits to the laboratory. 
On the occasion of each visit, blood pressure was measured by the same experienced observer using a standard mercury sphygmomanometer, taking the first and the fifth phases of Korotkoff sounds as systolic and diastolic values, respectively. Subjects were excluded if the average of the last two values obtained during each visit for systolic and diastolic blood pressures was greater than 139 and $89 \mathrm{mmHg}$, respectively.

\section{Maximal exercise test}

At the beginning of the study, exercised subjects underwent a maximal cycle ergometer exercise test, with $30 \mathrm{~W}$ increments every 3 min until exhaustion. Oxygen consumption and carbon dioxide were measured on a breath by breath basis, using a metabolic cart (Medical Graphics Corporation, CAD/NET 2001, St. Paul, MN), and peak oxygen consumption $\left(\mathrm{VO}_{2}\right.$ peak) was recorded as the highest value of oxygen consumption achieved during the test, calculated during an average 30-s interval.

\section{Study protocol}

Exercise protocols. Twelve subjects were submitted in a randomized order to three exercise trials performed at 30,50 or $70 \%$ of $\mathrm{VO}_{2}$ peak with an interval of at least 5 days. Each of the six possible combinations of exercise intensities was performed by two subjects. Exercise trials were conducted at the same time of day. Subjects were instructed not to exercise $48 \mathrm{~h}$ prior to the exercise trials, and to maintain similar activities and meal patterns.

For each exercise trial, subjects arrived at the laboratory between 1:00 and 3:00 p.m. Baseline blood pressure was measured every two and a half minutes in the sitting position for $20 \mathrm{~min}$. After this resting period, volunteers sat on a cycle ergometer (Mijnhardt, St. Paul, MN) and began the exercise, which consisted of 3 min of warm- up, $45 \mathrm{~min}$ of exercise at a workload to result in an intensity equivalent to 30,50 or $70 \%$ of $\mathrm{VO}_{2}$ peak, and 2 min of active recovery. After exercise, subjects rested in the sitting position for $90 \mathrm{~min}$. During exercise and throughout the recovery period, blood pressure was measured every $5 \mathrm{~min}$. Blood pressure was recorded by the same observer in all three exercise trials, using a standard mercury sphygmomanometer. Heart rate was monitored by electrocardiography (ECGTEB, SM300, São Paulo, SP) throughout the experimental protocol (resting to recovery), and was recorded simultaneously to blood pressure measurements. Baseline blood pressure and heart rate levels (average of 6 measurements after eliminating the first and last measurements of this period) were compared to the post-exercise levels, which were calculated for three recovery periods as the average of all measurements performed between 5 and 30 (R5-30), 35 and 60 (R35-60), and 65 and 90 (R65-90) min of recovery. The rate pressure product was determined as the product of heart rate and systolic blood pressure.

Exercise workloads were calculated based on oxygen consumption and workload during the maximal exercise test, using a linear regression equation. This procedure permitted us to calculate the workload corresponding to 30,50 , and $70 \%$ of $\mathrm{VO}_{2}$ peak. To verify the accuracy of exercise intensity, oxygen

Table 1 - Physical and cardiovascular characteristics of the subjects studied.

$\mathrm{BMI}=$ Body mass index; $\mathrm{SBP}=$ systolic blood pressure; $\mathrm{DBP}=$ diastolic blood pressure; $\mathrm{VO}_{2}$ peak = peak oxygen consumption.

\begin{tabular}{lrr} 
Variable & Exercised subjects & Rested subjects \\
\hline Age (years) & $22 \pm 0.7$ & $22 \pm 1.1$ \\
Weight $(\mathrm{kg})$ & $65.1 \pm 2.1$ & $66.6 \pm 2.7$ \\
Height $(\mathrm{cm})$ & $171 \pm 2.0$ & $171 \pm 2.0$ \\
$\mathrm{BMI}\left(\mathrm{kg} / \mathrm{m}^{2}\right)$ & $22.3 \pm 0.5$ & $22.9 \pm 1.0$ \\
Resting SBP $(\mathrm{mmHg})$ & $104 \pm 2.4$ & $109 \pm 2.3$ \\
Resting DBP $(\mathrm{mmHg})$ & $71 \pm 2.9$ & $68 \pm 1.9$ \\
VO $_{2}$ peak $\left(\mathrm{ml} \mathrm{kg}^{-1} \mathrm{~min}^{-1}\right)$ & $38.1 \pm 2.2$ & -
\end{tabular}


consumption was measured in all subjects at 15, 30 and $45 \mathrm{~min}$ of exercise (Medical Graphics Corporation, CAD/NET 2001) during the exercise trials.

Resting protocol. To determine any potential diurnal variations in blood pressure, 12 subjects performed a non-exercise control trial. During this trial, the subjects were submitted to the same experimental protocol as applied to the exercise trials, but instead of pedaling they rested in the sitting position for $45 \mathrm{~min}$. Blood pressure and heart rate were measured by the same procedures and at the same times as during the exercise trials.

\section{Statistical analysis}

Baseline levels in different exercise trials were analyzed by one-way analysis of variance for repeated measures. Blood pressure, heart rate and rate pressure product responses after exercise were evaluated by two-way analysis of variance (ANOVA) for repeated measures (BMDP, 1985, University of California, Los Angeles, CA), establishing exercise intensity (30, 50 and $70 \%$ of $\mathrm{VO}_{2}$ peak) and recovery stages (baseline, R5-30, R3560 and R65-90) as main factors. During the resting protocol, heart rate, blood pressure and rate pressure product were analyzed by one-way analysis of variance for repeated measures. When significance was found, Scheffé's test was employed. $\mathrm{P}<0.05$ was accepted as being statistically significant. Data are reported as mean \pm SEM.

\section{Results}

\section{Effect of exercise on cardiovascular responses}

Exercise intensity. Oxygen consumption measured during exercise trials at 30 and $50 \%$ of $\mathrm{VO}_{2}$ peak corresponded to $32.8 \pm 1.5$ and $53.9 \pm 1.6 \%$ of $\mathrm{VO}_{2}$ peak, respectively, while oxygen consumption during the exercise trial at $70 \%$ of $\mathrm{VO}_{2}$ peak corresponded to $80.1 \pm 2.1 \%$ of $\mathrm{VO}_{2}$ peak. Actually, these observations show that the exercise intensities studied were 30,50 and $80 \%$ of $\mathrm{VO}_{2}$ peak.

As expected, heart rate, rate pressure product, and systolic and mean blood pressures increased significantly during exercise and the increases were greater with higher exercise intensities. Diastolic blood pressure did not change during exercise of any intensity (Table 2).

Blood pressure. Systolic, mean and diastolic blood pressure values are shown in Figure 1. Baseline systolic, mean and diastolic blood pressures were similar in all three exercise trials, and systolic, mean, and diastolic blood pressure responses were similar during the recovery period of exercise performed at 30,50 and $80 \%$ of $\mathrm{VO}_{2}$ peak. Thus, systolic (marginal values - baseline $=$ $107.4 \pm 3.1$ vs R5-30 = 103.6 \pm 3.4 , R35-60 $=101.0 \pm 3.5$ and R65-90 = $102.5 \pm 3.4$ $\mathrm{mmHg}, \mathrm{P}<0.05$ ), mean (marginal values baseline $=85.6 \pm 3.0 v s \mathrm{R} 5-30=82.2 \pm 3.0$, $\mathrm{R} 35-60=80.8 \pm 3.2$ and $\mathrm{R} 65-90=82.2 \pm 3.1$ $\mathrm{mmHg}, \mathrm{P}<0.05$ ) and diastolic (marginal values - baseline $=74.7 \pm 3.1$ vs R5-30 $=71.6 \pm$ 3.0, R35-60 = 70.8 \pm 3.1 and $\mathrm{R} 65-90=72.2$ $\pm 3.0 \mathrm{mmHg}, \mathrm{P}<0.05$ ) blood pressure levels were significantly lower than baseline throughout the recovery period.

Heart rate. Heart rate values are shown in Figure 2. Baseline heart rate was similar in all three exercise trials. Exercise at $30 \%$ of $\mathrm{VO}_{2}$ peak provoked a significant decrease in heart rate at R35-60 (65.1 $\pm 2.3 \mathrm{bpm})$ and R65-90 (64.0 $\pm 2.0 \mathrm{bpm})$ when compared with baseline $(72.5 \pm 3.2 \mathrm{bpm})$. On the other hand, exercise at $50 \%$ of $\mathrm{VO}_{2}$ peak elicited a significant increase in heart rate at R5-30 $(75.9 \pm 2.4 \mathrm{bpm})$, but not at R35-60 (68.1 \pm $2.3 \mathrm{bpm})$ or R65-90 (66.8 $\pm 2.6 \mathrm{bpm})$ when compared with baseline $(69.4 \pm 2.0 \mathrm{bpm})$. After exercise at $80 \%$ of $\mathrm{VO}_{2}$ peak, heart rate responses at R5-30 (96.5 $\pm 2.1 \mathrm{bpm})$ and R35-60 (81.2 \pm 2.5 bpm) were significantly 
higher than baseline $(71.6 \pm 2.5 \mathrm{bpm})$, but not at R65-90 (75.8 $\pm 7.2 \mathrm{bpm})$ when they were similar to baseline.

Rate pressure product. Rate pressure product values are shown in Figure 3. Baseline rate pressure product was similar in all three exercise trials. Exercise at $30 \%$ of $\mathrm{VO}_{2}$ peak significantly decreased rate pressure product throughout the recovery period (baseline $=7930 \pm 314$ vs R5-30 $=7150 \pm$ $326, \mathrm{R} 35-60=6794 \pm 349, \mathrm{R} 65-90=6628 \pm$ $311, \mathrm{P}<0.05)$. Exercise at $50 \%$ of $\mathrm{VO}_{2}$ peak, however, caused no significant change in post-exercise rate pressure product (baseline $=7459 \pm 318 v s \mathrm{R} 5-30=7865 \pm 299, \mathrm{R} 35-60$ $=6873 \pm 323$, R65-90 = 6878 \pm 377 ). Exercise at $80 \%$ of $\mathrm{VO}_{2}$ peak provoked a significant increase in rate pressure product at R530 (baseline $=7468 \pm 267$ vs $\mathrm{R} 5-30=9818$ $\pm 366, \mathrm{P}<0.05)$, and no change at R35-60 $(7931 \pm 364)$ and R65-90 (7664 \pm 322$)$.

\section{Effect of resting on cardiovascular responses}

Blood pressure. During resting trial, systolic (baseline $=107.7 \pm 2.4$ vs R5-30 $=$ $106.2 \pm 3.1, \mathrm{R} 35-60=105.9 \pm 2.5$ and R65$90=106.0 \pm 2.2 \mathrm{mmHg}$ ) and mean (baseline $=80.8 \pm 1.9$ vs $\mathrm{R} 5-30=81.2 \pm 1.9, \mathrm{R} 35-60=$ $81.3 \pm 1.9$ and $\mathrm{R} 65-90=82.3 \pm 1.6 \mathrm{mmHg}$ ) blood pressures did not change significantly, while diastolic blood pressure was signifi- cantly higher than baseline $(67.2 \pm 2.0$ $\mathrm{mmHg})$ at $\mathrm{R} 65-90(70.3 \pm 2.1 \mathrm{mmHg}$, $\mathrm{P}<0.05$ ).

Heart rate. During the resting trial, heart rate did not change (baseline $=65.2 \pm 2.3 \mathrm{vs}$ $\mathrm{R} 5-30=64.5 \pm 2.4, \mathrm{R} 35-60=63.7 \pm 2.5$ and $\mathrm{R} 65-90=64.3 \pm 2.8 \mathrm{bpm}$ ).

Rate pressure product. Rate pressure product was also unchanged throughout the non-exercise control trial (baseline $=7027 \pm$ 303 vs R5-30 $=6832 \pm 269, \mathrm{R} 35-60=6748$ \pm 310 and $\mathrm{R} 65-90=6823 \pm 324)$.

\section{Discussion}

The main findings of the present study are: 1) exercise bouts performed at 30,50 and $80 \%$ of $\mathrm{VO}_{2}$ peak provoked similar postexercise hypotension in young normotensive humans; 2) exercise at $30 \%$ of $\mathrm{VO}_{2}$ peak, but not at 50 and $80 \%$ of $\mathrm{VO}_{2}$ peak, significantly decreased heart rate levels during the recovery period; 3 ) after exercise at $30 \%$ of $\mathrm{VO}_{2}$ peak, rate pressure product was lower than baseline levels, and 4) exercise at 50 and $80 \%$ of $\mathrm{VO}_{2}$ peak did not reduce postexercise rate pressure product.

The present data confirm the results obtained in previous studies $(1,5,7,8,10$ $12,15,16,18,19)$ showing that a single bout of steady-state exercise significantly decreases post-exercise blood pressure in nor-

\begin{tabular}{|c|c|c|c|c|c|c|}
\hline & \multicolumn{2}{|c|}{$30 \% \mathrm{VO}_{2}$ peak } & \multicolumn{2}{|c|}{$50 \% \mathrm{VO}_{2}$ peak } & \multicolumn{2}{|c|}{$80 \% \mathrm{VO}_{2}$ peak } \\
\hline & Baseline & Exercise & Baseline & Exercise & Baseline & Exercise \\
\hline SBP & $110 \pm 3$ & $124 \pm 3^{*+}$ & $107 \pm 3$ & $148 \pm 5^{*+}$ & $105 \pm 3$ & $167 \pm 5^{*+}$ \\
\hline MBP & $87 \pm 3$ & $91 \pm 3^{*+}$ & $86 \pm 3$ & $97 \pm 3^{*+}$ & $84 \pm 3$ & $105 \pm 3^{*+}$ \\
\hline DBP & $76 \pm 3$ & $75 \pm 3$ & $75 \pm 3$ & $71 \pm 3$ & $73 \pm 3$ & $74 \pm 3$ \\
\hline $\mathrm{HR}$ & $73 \pm 3$ & $101 \pm 4^{*+}$ & $69 \pm 2$ & $130 \pm 3^{*+}$ & $72 \pm 3$ & $170 \pm 3^{*+}$ \\
\hline $\mathrm{RP}$ & $7930 \pm 314^{+}$ & $12409 \pm 365^{*+}$ & $7459 \pm 318$ & $19211 \pm 550^{*+}$ & $7468 \pm 267$ & $28354 \pm 877^{*+}$ \\
\hline
\end{tabular}


motensive humans. The absence of a blood pressure fall during the non-exercise control trial shows that, in fact, the decreased blood pressure levels after exercise are due to the exercise effect and not to the normal diurnal blood pressure variations.

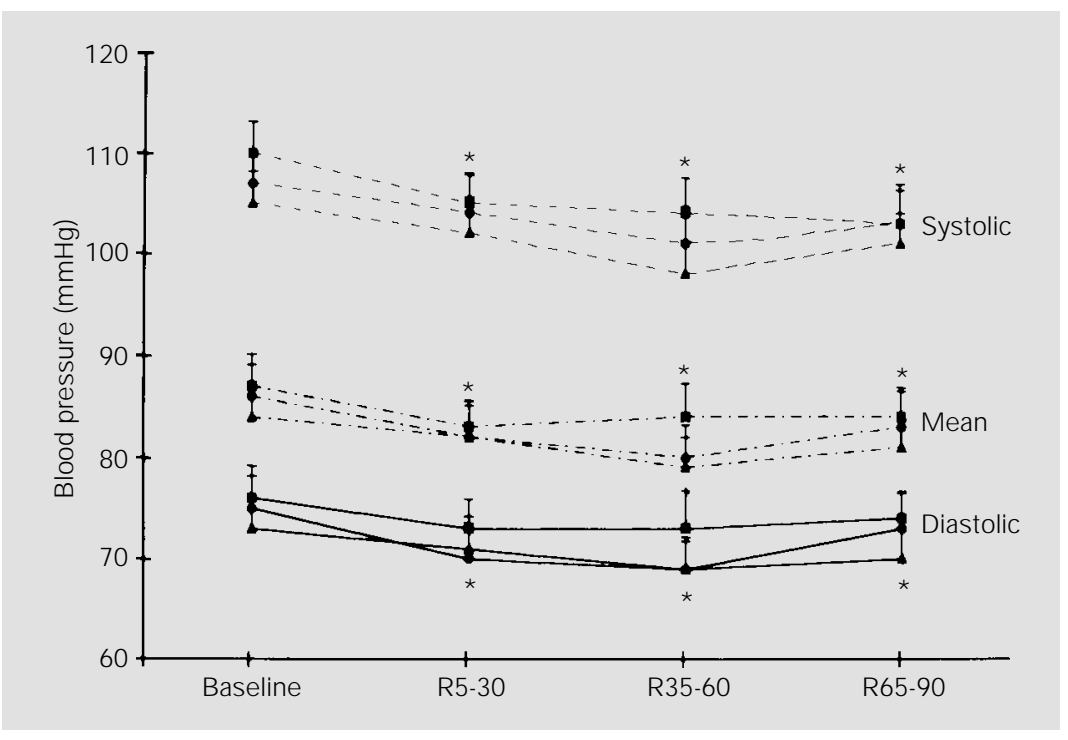

Figure 1 - Systolic, mean, and diastolic blood pressures at baseline and after exercise (R530 , mean value between 5 and 30 min; R35-60, mean value between 35 and 60 min; R6590, mean value between 65 and $90 \mathrm{~min}$ ) performed at 30 (filled squares), 50 (filled circles) and $80 \%$ (filled triangles) of $\mathrm{VO}_{2}$ peak. $* \mathrm{P}<0.05$ compared to baseline ( two-way analysis of variance for repeated measures and Scheffé's post-hoc test).

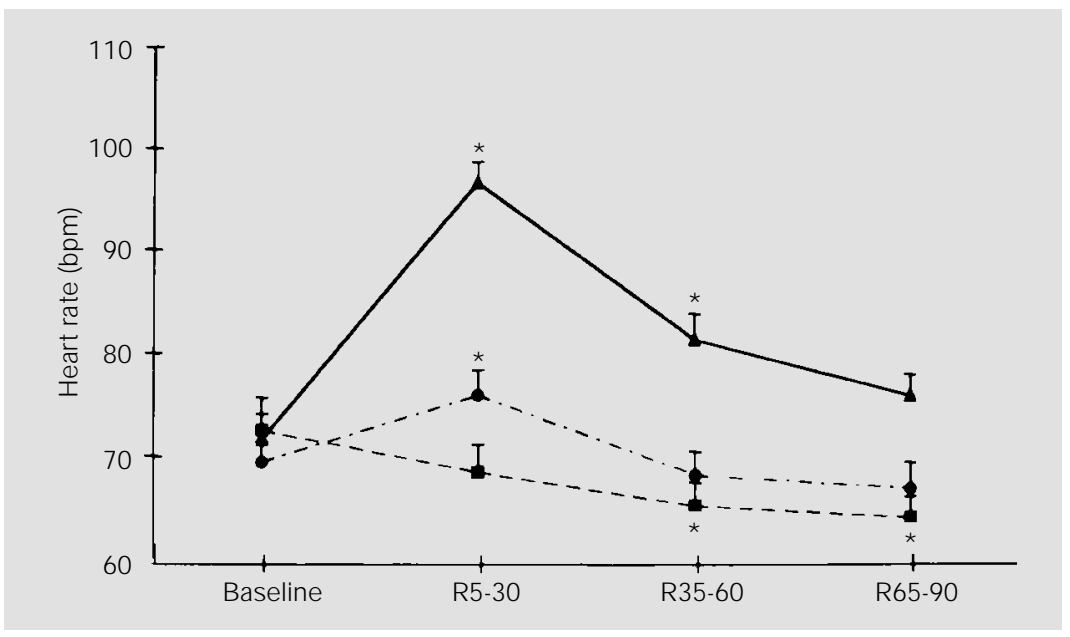

Figure 2 - Heart rate at baseline and after exercise (R5-30, mean value between 5 and 30 min; R35-60, mean value between 35 and 60 min; R65-90, mean value between 65 and 90 $\mathrm{min}$ ) performed at 30 (filled squares), 50 (filled circles) and $80 \%$ (filled triangles) of $\mathrm{VO}_{2}$ peak. $* \mathrm{P}<0.05$ compared to baseline (two-way analysis of variance for repeated measures and Scheffé's post-hoc test).
Since exercise intensity plays a role in hemodynamic, termoregulatory and neural responses during exercise $(22,23)$, we would expect that different exercise intensities would determine distinct post-exercise blood pressure responses. However, this was not the case in the present study, in which exercise performed at 30,50 and $80 \%$ of $\mathrm{VO}_{2}$ peak provoked similar hypotension during the recovery period. Pescatello et al. (14) did not observe any blood pressure fall after $30 \mathrm{~min}$ of exercise performed at $40 \%$ or $70 \%$ of $\mathrm{VO}_{2}$ peak, and Piepoli et al. (15) observed that maximal exercise, but not submaximal steady-state exercise, provoked a post-exercise blood pressure fall in young normotensive humans. In older hypertensives, Hagberg et al. (9) reported that hypotension was greater after exercise at $70 \%$ of $\mathrm{VO}_{2}$ peak than after exercise at $50 \%$ of $\mathrm{VO}_{2}$ peak. The contrast between the present study and others $(9,14,15)$ may be related to exercise duration or to the population investigated. In our study, normotensive humans were exercised for 45 min at all three intensities $(30,50$ and $80 \%$ of $\mathrm{VO}_{2}$ peak), while in the study of Pescatello et al. (14) normotensive humans were exercised for $30 \mathrm{~min}$, and in the study of Piepoli et al. (15) exercise duration was shorter during maximal than submaximal bouts. In fact, Bennett et al. (1) reported that a 10-min exercise period did not induce a post-exercise blood pressure fall in normotensives, but additional exercise periods (4 bouts of $10 \mathrm{~min}$ ) produced a significant decrease in blood pressure. Moreover, Overton et al. (13) observed that $40 \mathrm{~min}$ of exercise performed by spontaneously hypertensive rats provoked a greater and longer post-exercise hypotension than 20 min of exercise. Hagberg et al. (9) studied older hypertensive humans, while we investigated young normotensive humans. It is well understood that older subjects have vascular musculature alterations and decreased baroreceptor sensitivity (24) which may modify post-exercise hemodynamic responses. 
Previous studies on young normotensive humans $(1,4,5,7,8,10-12,14,15,18,19)$ have shown controversial results in regard to heart rate behavior during post-exercise period. The present results suggest that this controversy may be related to exercise intensity since we observed that low ( $30 \% \mathrm{VO}_{2}$ peak), but not moderate $\left(50 \% \mathrm{VO}_{2}\right.$ peak) intensity exercise provoked bradycardia during the recovery period. Moreover, high intensity exercise $\left(80 \% \mathrm{VO}_{2}\right.$ peak) provoked a transient tachycardia after exercise. In fact, previous studies showed a tendency toward a positive relationship between exercise intensity and post-exercise heart rate levels.

The concept that the product of systolic blood pressure and heart rate (i.e., rate pressure product) is well correlated to myocardium oxygen consumption in young healthy subjects (25) as well as in cardiac patients (26) has been well established. Some investigators have observed that angina pectoris occurs at a constant value of rate pressure product (27), and that the degree of STsegment depression is correlated with rate pressure product (28). Thus, the risk of cardiovascular problems increases with greater levels of rate pressure product. The present study demonstrated that an acute bout of low intensity exercise, besides producing lower increases in rate pressure product during exercise, also decreases post-exercise ratepressure product below resting levels. Hence it reduces myocardial oxygen consumption and, consequently, the cardiovascular risks after exercise. In contrast, moderate and high intensity exercise bouts produce greater increases in rate pressure product during exercise and fail to reduce rate pressure product below baseline during the recovery period. These findings, if reproducible in cardiac patients, may have clinical implications, especially in relation to exercise prescription in cardiac rehabilitation programs.

The present study does not provide explanations about the mechanism by which low intensity exercise simultaneously de- creases post-exercise blood pressure, heart rate and, consequently, rate pressure product in young normotensive subjects. Nevertheless, it is possible that low intensity exercise induces a reduction in sympathetic nerve activity to the heart and blood vessels during the recovery period. Chen et al. (3) observed a decrease in sympathetic tone to the heart after mild exercise in spontaneously hypertensive rats and Floras et al. (7) and Halliwill et al. (10) found a significant decrease in muscle sympathetic nerve activity after acute exercise in hypertensive and normotensive humans.

Despite the fact that both moderate and high intensity exercise decreased blood pressure, no reduction in rate pressure product was observed. Halliwill et al. (10) observed that after an acute bout of moderate exercise, baroreflex control of heart rate and blood vessels is distinctly regulated. In other words, sympathetic drive to the heart increases, while sympathetic drive to blood vessels decreases. Thus, it is possible that different exercise

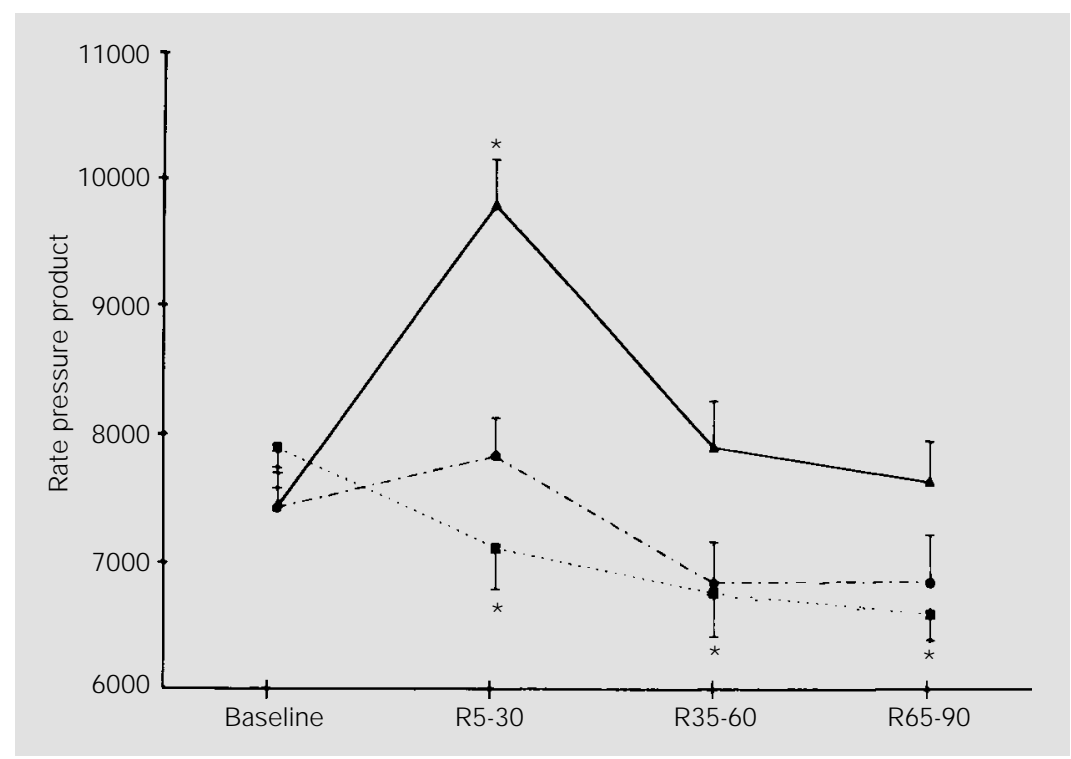

Figure 3 - Rate pressure product (heart rate $x$ systolic blood pressure) at baseline and after exercise (R5-30, mean value between 5 and 30 min; R35-60, mean value between 35 and $60 \mathrm{~min}$; R65-90, mean value between 65 and $90 \mathrm{~min}$ ) performed at 30 (filled squares), 50 (filled circles) and $80 \%$ (filled triangles) of $\mathrm{VO}_{2}$ peak. $* \mathrm{P}<0.05$ compared to baseline (twoway analysis of variance for repeated measures and Scheffé's post-hoc test). 
intensities may distinctly affect the baroreflex control of heart rate, but not the baroreflex control of blood vessels. Moreover, the vasodilatory response (15) and/or the decrease in blood volume (13) may play a role in the post-exercise blood pressure fall. It is well understood that muscle metabolites and heat accumulation are directly related to exercise intensity, and sweating rate is greater during more intense exercise (29). In addition, the increased local muscle metabolites $(30,31)$ and/or heat production (8) are also potential stimuli for the increased heart rate responses after moderate and high intensity exercise.

Limitations. We studied the impact of acute exercise performed at 30,50 and 80\% of $\mathrm{VO}_{2}$ peak on cardiovascular responses up to 90 min of recovery in young healthy subjects. Thus, we do not know whether the decreased rate pressure product after low intensity exercise persists longer than 90 min or occurs in the presence of certain cardiovascular disturbances such as hypertension, myocardial ischemia or even heart failure. Future studies, however, should address the effect of varying intensity of exercise on post-exercise cardiovascular responses in patients with cardiovascular disturbances.

We studied exercise at three different intensities (30, 50 and $80 \%$ of $\mathrm{VO}_{2}$ peak). It is possible that other intensities could have distinct effects on post-exercise cardiovascular responses.

In conclusion, in young normotensive humans, varying the intensity of exercise from 30 to $80 \%$ of $\mathrm{VO}_{2}$ peak did not influence the magnitude of post-exercise hypotension. However, in contrast to exercise at 50 and $80 \%$ of $\mathrm{VO}_{2}$ peak, exercise at $30 \%$ of $\mathrm{VO}_{2}$ peak significantly decreased rate pressure product during the recovery period.

\section{Acknowledgments}

We gratefully acknowledge the volunteers involved in this study. We also thank our laboratory team for technical assistance.

\section{References}

1. Bennett T, Wilcox RG \& MacDonald IA (1984). Post-exercise reduction of blood pressure in hypertensive men is not due to acute impairment of baroreflex function. Clinical Science, 67: 97-103.

2. Boone J B, Probst MM, Rogers $M W \&$ Berger R (1993). Postexercise hypotension reduces cardiovascular responses to stress. J ournal of Hypertension, 11: 449453.

3. Chen Y, Chandler MP \& DiCarlo SE (1995). Acute exercise attenuates cardiac autonomic regulation in hypertensive rats. Hypertension, 26: 676-683.

4. Cléroux J, Kouamé $N$, Nadeau $A$, Coulombe D \& Lacourcière Y (1992). Aftereffects of exercise on regional and systemic hemodynamics in hypertension. Hypertension, 19: 183-191.

5. Coats AJ S, Conway J, Isea J E, Pannarale G, Sleight P \& Somers VK (1989). Systemic and forearm vascular resistance changes after upright bicycle exercise in man. J ournal of Physiology, 413: 289-298.

6. Collins HL \& DiCarlo SE (1993). Attenua- tion of postexertional hypotension by cardiac afferent blockade. American J ournal of Physiology, 265: H1179-H1183.

7. Floras J S, Sinkey CA, Aylward PE, Seals DR, Thorén PN \& Mark AL (1989). Post exercise hypotension and sympathoinhibition in borderline hypertensive men. Hypertension, 14: 28-35.

8. Franklin PJ , Green DJ \& Cable NT (1993). The influence of thermoregulatory mechanisms on post-exercise hypotension in humans. J ournal of Physiology, 470: 231241.

9. Hagberg J M, Montain SJ \& Martin WH (1987). Blood pressure and hemodynamic responses after exercise in older hypertensives. J ournal of Applied Physiology, 63: 270-276.

10. Halliwill JR, Taylor JA \& Eckberg DL (1996). Impaired sympathetic vascular regulation in humans after acute dynamic exercise. J ournal of Physiology, 495: 279288.

11. Hara K \& Floras J S (1994). Influence of naloxone on muscle sympathetic nerve activity, systemic and calf haemodynamics and ambulatory blood pressure after exercise in mild essential hypertension. J ournal of Hypertension, 13: 447-461.

12. Kaufman FL, Hughson RL \& Schaman J P (1987). Effect of exercise on post-exercise blood pressure in normotensive and hypertensive subjects. Medicine and Science in Sports and Exercise, 19: 17-20.

13. Overton J M, J oyner MJ \& Tipton CM (1988). Reductions in blood pressure after acute exercise by hypertensive rats. J ournal of Applied Physiology, 64: 748752.

14. Pescatello LS, Fargo AE, Leach J r CN \& Scherzer HH (1991). Short-term effect of dynamic exercise on arterial blood pressure. Circulation, 83: 1557-1561.

15. Piepoli M, Isea JE, Pannarale G, Adamopoulos S, Sleight P \& Coats AJ S (1994). Load dependence of changes in forearm and peripheral vascular resistance after acute leg exercise in man. J ournal of Physiology, 478: 357-362.

16. Raglin J S, Turner PE \& Eksten F (1993). 
State anxiety and blood pressure following $30 \mathrm{~min}$ of leg ergometry or weight training. Medicine and Science in Sports and Exercise, 25: 1044-1048.

17. Shyu BC \& Thorén P (1976). Circulatory events following spontaneous muscle exercise in normotensive and hypertensive rats. Acta Physiologica Scandinavica, 128: 515-524.

18. Somers VK, Conway J, Coats A, Isea J \& Sleight P (1991). Postexercise hypotension is not sustained in normal and hypertensive humans. Hypertension, 18: 211215.

19. Wilcox RG, Bennett T, Brown AM \& MacDonald IA (1982). Is exercise good for high blood pressure? British Medical J ournal, 285: 767-769.

20. Convertino VA \& Adams WC (1991). Enhanced vagal baroreflex response during 24 hours after acute exercise. American J ournal of Physiology, 260: R570-R575.

21. Kenney MJ \& Seals DR (1993). Postexercise hypotension: key features, mechanisms, and clinical significance. Hyperten- sion, 22: 653-664.

22. Leuenberger U, Sinoway L, Gubin S, Gaul L, Davis D \& Zelis R (1993). Effects of exercise intensity and duration on norepinephrine spillover and clearance in humans. J ournal of Applied Physiology, 75: 668-674.

23. Saito $M$, Tsukanaka A, Yanagihara $D \&$ Mano T (1993). Muscle sympathetic nerve responses to graded leg cycling. J ournal of Applied Physiology, 75: 663-667.

24. Ebert TJ , Morgan BJ , Barney J A, Denahan T \& Smith J J (1992). Effects of aging on baroreflex regulation of sympathetic activity in humans. American J ournal of Physiology, 263: H798-H803.

25. Kitamura K, J orgensen CR, Gobel FL, Taylor HL \& Wang Y (1972). Hemodynamic correlates of myocardial oxygen consumption during upright exercise. J ournal of Applied Physiology, 32: 516-522.

26. Gobel FL, Nordstrom LA, Nelson RR, J orgensen CR \& Wang Y (1978). The rate pressure product as an index of myocardial oxygen consumption during exercise in patients with angina pectoris. Circulation, 57: 549-556.

27. Robinson BF (1967). Relation of heart rate and systolic blood pressure to the onset of pain in angina pectoris. Circulation, 1073-1083.

28. Dentry J M, Piette RJ F \& Brasseur LA (1970). Hemodynamic determinants of exercise ST-segment depression in coronary patients. Circulation, 42: 593-599.

29. McArdle WD, Katch FI \& Katch VL (1996). Exercise and thermal stress. In: Exercise Physiology: Energy, Nutrition, and Human Performance. Williams \& Wilkins, Baltimore, MD.

30. Costa F \& Biaggioni I (1994). Role of adenosine in the sympathetic activation produced by isometric exercise in humans. J ournal of Clinical Investigation, 93: 16541660.

31. Middlekauff HR, Nitzsche EU, Nguyen AH $\&$ Hoh CK (1997). Modulation of renal cortical blood flow during static exercise in humans. Circulation Research, 80: 62-68. 